Research Paper

\title{
A Bottleneck in Understanding Metastatic Cancer Stem Cell of Peritoneal Seeding from Gastric Cancer: A Null Result in Brief
}

\author{
Xin-Zu Chen ${ }^{1}$, Xiao-Hai Song ${ }^{1 *}$, Xiao-Long Chen ${ }^{1 *}$, Chang-Chun Li ${ }^{1}$, Xian-Ming Mo², Jian-Kun Hu${ }^{1 凶}$ \\ 1. Department of Gastrointestinal Surgery and Laboratory of Gastric Cancer, State Key Laboratory of Biotherapy, West China Hospital, Sichuan University, \\ Chengdu, China: \\ 2. Laboratory of Stem Cell Biology, West China Hospital, Sichuan University, Chengdu, China. \\ *Xiao-Hai Song and Xiao-Long Chen contributed equally in this work as co-first authors. \\ $\square$ Corresponding author: Prof. Jian-Kun Hu, M.D., Ph.D., F.R.C.S., Department of Gastrointestinal Surgery, West China Hospital, Sichuan University, Guo Xue \\ Xiang 37, Chengdu 610041, Sichuan Province, China. Email: hujkwch@126.com \\ (C) Ivyspring International Publisher. This is an open access article distributed under the terms of the Creative Commons Attribution (CC BY-NC) license \\ (https://creativecommons.org/licenses/by-nc/4.0/). See http://ivyspring.com/terms for full terms and conditions.
}

Received: 2017.06.28; Accepted: 2017.08.30; Published: 2017.09.16

\begin{abstract}
The capture of peritoneal metastatic cancer stem cell of human gastric cancer (pMCSC-hGC) is important to further understand the mechanism of peritoneal metastasis in gastric cancer patients. Previously, cancer stem cells (CSCs) of gastric and rectal cancers were captured and identified. However, the bottleneck of capturing PMCSC-hGC may be the scarce surgical specimen and limited volume of peritoneal metastatic lesions from gastric cancer. Only $5.2 \%$ of patients were diagnosed of unpredictive peritoneal seeding intraoperatively, while none cell sphere were successfully formed through the identical culture approach based on peritoneal metastatic nodules. The attempt to enrich and capture pMCSC of transplanted gastric cancer (pMCSC-tGC) in immunodeficiency mice model through intraperitoneal injection of CSC-hGC may be a considerable and feasible alteration.
\end{abstract}

Key words: gastric cancer; peritoneal metastasis; cancer stem cell; clonal heterogeneity; null result

\section{Introduction}

Gastric cancer is still one of the leading causes of cancer-related death in China [1]. The overall effectiveness of screening and treatment is not improved apparently during the first decade of the 21 th century [2]. Large proportion of locally advanced or metastatic gastric cancers $(>80 \%)$ attributes to the poor prognosis in Chinese population [3, 4]. Peritoneal seeding is a common pattern of distal metastasis from gastric cancer, and is associated with incurability and fairly limited survival time [5].

The biological procedures from the migration of primary cancer cell to the homing of metastatic cancer cells in peritoneum might be different from circulatory metastasis or direct invasion. The hypoxia environment and the mesothelial cell layer of peritoneal cavity would be the unique conditions for peritoneal seeding against the circulatory metastasis. Epithelial-mesenchymal transition (EMT) may induce the migration of CSCs and becomes an important mechanism of metastasis [6]. The EMT and mesenchymal-epithelial transition (MET) in peritoneal metastasis might be different or simplied, compared with the circulatory metastasis.

Clonal heterogeneity exists in the homeostasis of epithelial cancer, and it may be induced by the mutation acquisition and environmental changes to initial cancer stem cell (CSC) [7]. We hypothesized the CSCs might be also heterogeneous in a tumor, either hereditary or acquired. Those CSCs with the capacity of metastasis could be classified as a cluster of metastatic cancer stem cells (MCSCs). Circulatory MCSCs (cMCSC) have been captured in peripheral 
blood of solid tumor patients, such as gastric cancer [8]. However, till now, we didn't find a report of capturing and identifying peritoneal MCSCs of human gastric cancer (pMCSC-hGC) in the literatures of the PubMed database.

Previously, we have already captured and identified the cancer stem cell-like cells of human gastric cancer (CSC-hGC) and rectal cancer (CSC-hRC) from tumor tissues, and get successful subculture for subsequent experiments [8-10]. CSC-hGC and CSC-hRC carrying CD44 and CD54 surface markers, the putative biomakers of CSCs [11], had the capacity of self-renewal and differentiation to epithelial cells. Compared to circulatory metastasis, cancerous nodules of peritoneal metastasis may have similar features of primary solid tumors. Thus, we designed to capture the pMCSC-hGC through the identical methods $[8,9,12]$, in order to subsequently identify the difference of biological behaviors between pMCSC-hGC and CSC-hGC.

\section{Materials and Methods}

\section{Specimen collection}

The surgical patients with gastric cancer in West China Hospital, Sichuan University were assessed about the eligibility during the 2014. If the preoperative radiology indicated intuitional and even bulky peritoneal metastasis $(\mathrm{cP}+)$, the patients were excluded because of inability to collect seeding tumors. In this case, the chemotherapy was recommended instead of laparotomy or laparoscopy. Those patients with predictively resectable diseases (cTxNxM0) were given laparotomy or laparoscopy. Only occasional and unpredictive findings of peritoneal metastasis were eligible to collect the peritoneal seeding nodules intraoperativley. The classification system of peritoneal metastasis in gastric cancer patients was described elsewhere [5]. After the confirmation of adenocarcinoma involved through the intraoperative frozen section, the suspicious nodules $(\leq 3)$ were additionally dissected from parietal peritoneum, mesentery or omentum without major iatrogenic injury. The fat tissues were picked up from the harvested cancerous nodules in vitro as completely as possible.

\section{Cell culture}

The methods to capture pMCSC-hGC were specified elsewhere idential to CSC-hGC [8]. In this experiment, the harvested cancerous nodules were washed 5 times using PBS with 10\% Penicillin-Streptomycin solution (HyClone, US). The specimens were cut into pieces and digested with IV collagenase for $30-60$ minutes in the incubator at $37^{\circ} \mathrm{C}$. Cell suspension were collected after filtered by 70-um filter. After centrifugation with a speed of $1000 \mathrm{r} / \mathrm{min}$ for 5 minutes, cell sedimentation was collected and cultured in serum-free medium in $\mathrm{CO}_{2}$ incubator at $37^{\circ} \mathrm{C}$. After 3-4 weeks, if some cancer cells grew to form spheres in culture, it was considered that pMCSC-hGCs might be enriched and identification was further required.

\section{Ethics}

This investigation was approved by the Biomedical Ethical Committee of West China Hospital [8], experiments were performed in accordance with the principles of Declaration of Helsinki. All the surgical patients were preoperatively given informed consent on tissue donation in scientific investigations.

\section{Results}

In the 2014, in total 192 gastric cancer patients were operated in a surgical group in West China Hospital. Ten patients (5.2\%) were diagnosed of unpredictive peritoneal seeding intraoperatively, classified as $2 \mathrm{P} 1$ diseases and 8 miliary $\mathrm{P} 2 / \mathrm{P} 3$ diseases (Figure 1). Those miliary P2/P3 diseases were eligible to collect peritoneal or omental cancerous nodules intraoperatively. The median survival time of this group was 6.5 (range 3-13) months. Digestion of peritoneal cancerous nodules and culture was performed in the eight cases. However, we finally failed to form cell sphere through the identical culture approach in the patients with peritoneal seeding (Table 1). These null results made the attempt to capture pMCSC-hGC interrupted.

Table 1. Basic information of patients with peritoneal seeding (miliary P2/P3).

\begin{tabular}{|c|c|c|c|c|c|c|c|c|}
\hline Patients & Hospitalization ID & Sex & Age (y) & Site & TNM stage & Grade & Survival time (m) & Sphere formation \\
\hline 1 & 0013525222 & Male & 44 & UML & $\mathrm{sT} 4 \mathrm{aN}+\mathrm{M} 1, \mathrm{pP} 3$ & G3 & 5 & No \\
\hline 2 & 0013550174 & Male & 65 & $\mathrm{~L}$ & $\mathrm{sT} 4 \mathrm{aN}+\mathrm{M} 1, \mathrm{pP} 2$ & Gx & 6 & No \\
\hline 3 & 0011894353 & Male & 72 & $\mathrm{U}$ & sT4aN+M1, pP3 & G2 & 7 & No \\
\hline 4 & 0014430396 & Male & 43 & UML & $\mathrm{sT} 4 \mathrm{bN}+\mathrm{M} 1, \mathrm{pP} 3$ & Gx & 6 & No \\
\hline 5 & 0014491603 & Female & 63 & UML & $\mathrm{sT} 4 \mathrm{bN}+\mathrm{M} 1, \mathrm{pP} 3$ & G3 & 7 & No \\
\hline 6 & 0014447629 & Male & 68 & UM & sT4aN+M1, pP2 & $\mathrm{Gx}$ & 3 & No \\
\hline 7 & 0014672251 & Female & 68 & $\mathrm{M}$ & pT4bN3bM1, pP2 & G3 & 13 & No \\
\hline 8 & 0000428648 & Male & 41 & ML & sT4aN+M1, pP3 & Gx & 12 & No \\
\hline
\end{tabular}



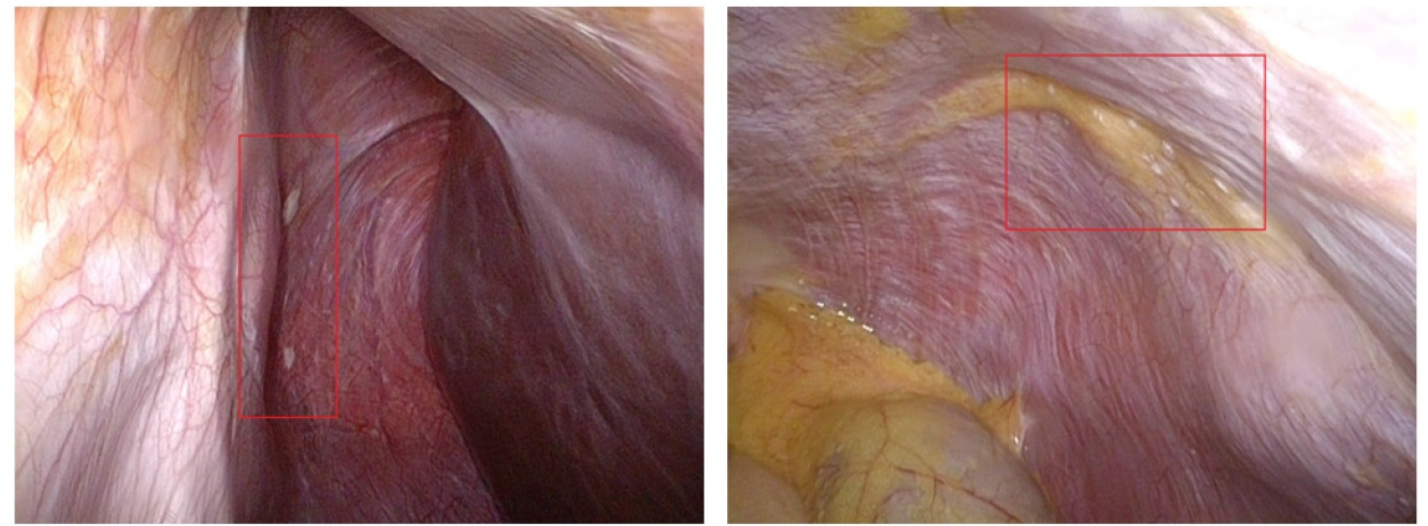

Figure 1. Miliary seeding tumors of gastric cancer patients.

Table 2. The cancer stem cell-based hypotheses of peritoneal metastasis from gastric cancer.

\begin{tabular}{|c|c|c|c|c|}
\hline Status & Site / stage & Cells & Steps & Potential mechanisms \\
\hline \multirow[t]{2}{*}{ Primary cancer of stomach } & Mucosa / T1 & CSC cluster & Step 1 & Hypothesis of "apoptosis remission" \\
\hline & Serosa / T4 & CSC high-invasive & Step 2 & Hypothesis of "EMT" \\
\hline \multirow[t]{2}{*}{ Free cancer cells or spheres } & $\begin{array}{l}\text { Peritoneal cavity / M1, CY1 } \\
\text { (hypoxia enviroment) }\end{array}$ & pMCSC & Step 3 & $\begin{array}{l}\text { Hypothesis of "seed" } \\
\text { Hypothesis of "EMT" }\end{array}$ \\
\hline & & pMCSC & Step 4 & Hypothesis of "anoikis" \\
\hline \multirow[t]{3}{*}{ Seeding cancer of peritoneum } & $\begin{array}{l}\text { Peritoneum / M1, P1 } \\
\text { (mesothelial cell layer) }\end{array}$ & pMCSC & Step 5 & $\begin{array}{l}\text { Hypothesis of "soil" } \\
\text { Hypothesis of "homing" } \\
\text { Hypothesis of "MET" }\end{array}$ \\
\hline & & pMCSC & Step 6 & $\begin{array}{l}\text { Hypothesis of "niche" } \\
\text { Hypothesis of "dormancy" }\end{array}$ \\
\hline & Adjacent organs / M1, P1 & pMCSC & Step 7 & Hypothesis of "EMT" \\
\hline
\end{tabular}

Abbreviations: CSC, cancer stem cell; EMT, epithelial-mesenchymal transition; MET, mesenchymal-epithelial transition; pMCSC, peritoneal metastatic cancer stem cell.

\section{Discussion}

To our knowledge, this was the first report on the attempt to capture pMCSC-hGCs. However, the null results made our attempt to capture pMCSC-hGC interrupted, although the original methods were able to capture CSC-hGC and CSC-hRC. Therefore, we reported our experiences with null results in brief, in order to draw attention to recognize the difficulty in capturing pMCSC-hGCs.

Certain theoretical and practical difficulties in achieving the capture need consider. With the improvement of the precise preoperative staging through radiological approaches, the most of the gastric cancer patients with bulky peritoneal seeding can be diagnosed and prevented from a unnecessary laparotomy. Although P2/P3 diseases were found in 8 patients, the cancerous lesions were shaped as miliary and minor (Figure 1). The volume of collected peritoneal or omental nodules was quite limited. Additionally, the collected tumor tissues were more likely to be contaminated with adjacent non-cancerous tissues. Therefore, the proportion of pMCSC-hGC was speculated to be less than that of CSC-hGC, as it should be a bottleneck of capturing and further understanding pMCSC-hGC.
The basic theory of peritoneal seeding should be the seed-soil hypotheses [13-15]. First, the high-invasive subpopulation of CSCs may penetrate the serosa through EMT machenism and become free and potential pMCSCs in the peritoneal cavity. Before homing in the peritoneum, the anoikis may happen in the free cancel cells to select pMCSC survivors, which are even able to protect the non-stem cells [16-18]. Compared to circulatory spread of cMCSCs, the EMT procedure was simplied in the peritoneal metastasis, from dropping off into the hypoxia environment to direct cross-talk with the micro-environment of peritoneum. The cadherin switch phenomenon is the principal molecular event in the EMT [19, 20], and may appear opposite at the beginning and the ending of peritoneal metastais. Therefore, it was supposed that the peritoneal metastasis of gastric cancer might be multistep and several mechanisms might be potentially involved in the whole procedure (Table 2 ) [21, 22].

Alternatively, we hypothesize it is possible to enrich the pMCSC via a transplanted tumor model, i.e. intraperitoneal injection of CSC-hGC in immunodeficiency mice. In this case, the transplanted intraperitoneal tumors may have greater volume of lesions and higher proportion of pMCSCs. Therefore, 
the pMCSC of transplanted gastric cancer (pMCSC-tGC) may probably be captured through identical culture approach and cell sphere formation. Continuous intraperitoneal injection of the first-generation pMCSC-tGCs in the animal model can judge the capacity of peritoneal seeding, and also harvest the second-generation pMCSC-tGCs or more generations. Comparisons of the cell behaviors and biology between pMCSC-tGC and primary CSC-hGC are able to help us with understanding the mechanisms of peritoneal metastasis from gastric cancer.

In a short, the capture of pMCSC-hGC is important to further understand the mechanism of peritoneal metastasis in gastric cancer patients. However, the bottleneck may be the scarce surgical specimen and limited volume of peritoneal metastatic lesions from gastric cancer. The attempt to capture pMCSC-tGC will be a considerable and feasible alteration.

\section{Compliance with Ethical Standards}

This investigation was granted by the National Natural Science Foundation of China (NSFC, no. 81301866), and the Outstanding Young Scientific Scholarship Foundation of Sichuan University, from the Fundamental Research Funds for the Central Universities of China (No. 2015SCU04A43). The funders did not play any role in the conception, analyses and writing. This investigation entitled as, Capture of metastatic cancer stem cell in peritoneal metastasis of gastric cancer and its mechanism of "epithelial-mesenchymal transition", was approved by the Biomedical Ethical Committee of West China Hospital, Sichuan University in the 2013. Informed consent for the storage and usage of surgical specimens in anonymous experiments was obtained preoperatively, from all individual gastric cancer patients included in this pilot study.

\section{Acknowledgements}

The National Natural Science Foundation of China (NSFC, no. 81301866), the Outstanding Young Scientific Scholarship Foundation of Sichuan University, from the Fundamental Research Funds for the Central Universities of China (No. 2015SCU04A43), and the Scientific Research Program from the Sichuan Provincial Health and Family Planning Commission (No. 16PJ362).

\section{Competing Interests}

The authors have declared that no competing interest exists.

\section{References}

1. Wang R, Chen XZ. High mortality from hepatic, gastric and esophageal cancers in mainland China: 40 years of experience and development. Clinics and research in hepatology and gastroenterology. 2014; 38: 751-6.

2. Chen XZ, Liu Y, Wang R, Zhang WH, Hu JK. Improvement of cancer control in mainland China: epidemiological profiles during the 2004-10 National Cancer Prevention and Control Program. Lancet. 2016; 388: S40.

3. Yang K, Choi YY, Zhang WH, Chen XZ, Song MK, Lee J, et al. Strategies to improve treatment outcome in gastric cancer: a retrospective analysis of patients from two high-volume hospitals in Korea and China. Oncotarget. 2016; 7: 44660-75.

4. Chen $\mathrm{XZ}$, Zhang $\mathrm{WH}, \mathrm{Hu} \mathrm{JK}$. A difficulty in improving population survival outcome of gastric cancer in mainland China: low proportion of early diseases. Medical oncology (Northwood, London, England). 2014; 31: 315.

5. Yang $\mathrm{K}$, Liu $\mathrm{K}$, Zhang WH, Lu ZH, Chen XZ, Chen XL, et al. The value of palliative gastrectomy for gastric cancer patients with intraoperatively proven peritoneal seeding. Medicine. 2015; 94: e1051.

6. Radisky DC, LaBarge MA. Epithelial-mesenchymal transition and the stem cell phenotype. Cell Stem Cell. 2008; 2: 511-2.

7. Donati G, Watt FM. Stem cell heterogeneity and plasticity in epithelia. Cell Stem Cell. 2015; 16: 465-76.

8. Chen $\mathrm{T}$, Yang $\mathrm{K}, \mathrm{Yu}$ J, Meng $\mathrm{W}$, Yuan $\mathrm{D}, \mathrm{Bi} \mathrm{F}$, et al. Identification and expansion of cancer stem cells in tumor tissues and peripheral blood derived from gastric adenocarcinoma patients. Cell Res. 2012; 22: 248-58.

9. Fan CW, Chen T, Shang YN, Gu YZ, Zhang SL, Lu R, et al. Cancer-initiating cells derived from human rectal adenocarcinoma tissues carry mesenchymal phenotypes and resist drug therapies. Cell Death Dis. 2013; 4: e828.

10. Zhang S, Shang Y, Chen T, Zhou X, Meng W, Fan C, et al. Human circulating and tissue gastric cancer stem cells display distinct epithelial-mesenchymal features and behaviors. J Cancer Res Clin Oncol. 2017: doi: 10.1007/s00432-017-2417-3. [Epub ahead of print].

11. Chen XL, Chen XZ, Wang YG, He D, Lu ZH, Liu K, et al. Clinical significance of putative markers of cancer stem cells in gastric cancer: A retrospective cohort study. Oncotarget. 2016; 7: 62049-69.

12. Man YG, Wang J, Jiang B, Mason J, Mannion C, Fu S, et al. Can current cell sorting methods capture cancer invasion and metastasis-causing stem cells? New Approaches combating Cancer \& Aging. 2014; 1: 1-28.

13. Togo S, Shimada H, Kubota T, Moossa AR, Hoffman RM. "Seed" to "soil" is a return trip in metastasis. Anticancer Res. 1995; 15: 791-4.

14. Herawi M, Leppert JT, Thomas GV, De Kernion JB, Epstein JI. Implants of noninvasive papillary urothelial carcinoma in peritoneum and ileocolonic neobladder: support for "seed and soil" hypothesis of bladder recurrence. Urology. 2006; 67: 746-50.

15. Yeung TL, Leung CS, Yip KP, Au Yeung CL, Wong ST, Mok SC. Cellular and molecular processes in ovarian cancer metastasis. A Review in the Theme: Cell and Molecular Processes in Cancer Metastasis. Am J Physiol Cell Physiol. 2015; 309: C444-56.

16. Frisch SM. E1a induces the expression of epithelial characteristics. J Cell Biol. 1994; 127: 1085-96.

17. Cao Z, Livas T, Kyprianou N. Anoikis and EMT: Lethal "Liaisons" during Cancer Progression. Crit Rev Oncog. 2016; 21: 155-68.

18. Kim SY, Hong SH, Basse PH, Wu C, Bartlett DL, Kwon YT, et al. Cancer Stem Cells Protect Non-Stem Cells From Anoikis: Bystander Effects. J Cell Biochem. 2016; 117: 2289-301.

19. Cavallaro U, Schaffhauser B, Christofori G. Cadherins and the tumour progression: is it all in a switch? Cancer Lett. 2002; 176: 123-8.

20. Wheelock MJ, Shintani Y, Maeda M, Fukumoto Y, Johnson KR. Cadherin switching. J Cell Sci. 2008; 121: 727-35.

21. Chen XZ, Yang K, Hu JK, Mo XM. Hypotheses of potential mechanisms of cancer stem cell in peritoneal metastasis of gastric cancer. Chin J Bases Clin General Surg. 2014; 21: 772-7. [In Chinese].

22. Mannion C, Mason J, Hsiao YH, Wang J, Jiang B, Fu S, et al. A morphologically identifiable precursor of metastatic cancer. New Approaches combating Cancer \& Aging. 2016; 3: 1-8. 